\title{
PREVISÃO DE DEMANDA INTERMITENTE: UMA INVESTIGAÇÃO EMPÍRICA NA CADEIA DE SOBRESSALENTES DA MARINHA DO BRASIL
}

\author{
Leonardo Câmara de Araujo da Fonseca \\ Centro de Instrução e Adestramento Almirante Newton Braga (CIANB) \\ Av. Brasil, 10.500 - Olaria - Rio de Janeiro - RJ \\ leonardo.camara@marinha.mil.br \\ Ricardo França Santos \\ Universidade Federal do Rio de Janeiro (UFRJ) \\ Av. Aluizio da Silva Gomes, 50 - Novo Cavaleiro - Macaé - RJ \\ ricardofranca@macae.ufrj.br \\ Thiago Fernandes Lima \\ Marinha do Brasil \\ Ilha das Cobras, s/n - Ed. Almirante Gastão Motta - Centro - Rio de Janeiro - RJ \\ fernandes.lima@marinha.mil.br \\ Wagner Correia de Souza \\ Marinha do Brasil \\ Ilha das Cobras, s/n - Ed. Almirante Gastão Motta - Centro - Rio de Janeiro - RJ \\ w.souza@marinha.mil.br
}

\begin{abstract}
RESUMO
Este artigo tem o propósito de identificar métodos de previsão de demanda intermitente mais adequados aos itens de sobressalentes da Marinha do Brasil, com o intuito de contribuir com o projeto de modernização do Sistema de Informações Gerenciais do Abastecimento (SINGRA), atualmente em curso. Assim, realizou-se uma comparação entre os métodos utilizados atualmente e os métodos Amortecimento Exponencial Simples (AES), Croston e Syntetos-Boylan Approximation (SBA). O Erro Escalonado Médio Absoluto foi o parâmetro utilizado nesta comparação, que se baseou em observações de uma série histórica de 30 trimestres para 276 itens de sobressalentes com característica de demanda intermitente, concluindo-se que os métodos SBA e AES são mais precisos que a sistemática atual.

Palavra-chave: Marinha do Brasil; Demanda Intermitente; Previsão de Demanda; Métodos de Previsão de Demanda; Peças de reposição.
\end{abstract}

\begin{abstract}
The present work intends to identify intermittent demand forecasting methods best suited to spare parts of the Brazilian Navy, in order to contribute to the modernization project of the Sistema de Informações Gerenciais do Abastecimento (SINGRA), currently underway. Thus, a comparison was made between the methods currently used and the Simple Exponential Smoothing (SES), Croston and Syntetos-Boylan Approximation (SBA) methods. The Mean Absolute Scaled Error was the parameter used in this comparison, which was based on
\end{abstract}


observations from a 30 quarter historical series of 276 spare parts items with intermittent demand feature, concluding that SBA and SES methods are more accurate than the one in use. Keywords: Brazilian Navy; Intermittent demand; Demand Forecasting; Demand Forecasting Methods; Spare parts.

\section{Como Citar:}

FONSECA, L. C. A. et al. Previsão de demanda intermitente: Uma investigação empírica na cadeia de sobressalentes da Marinha do Brasil. In: SIMPÓSIO DE PESQUISA OPERACIONAL E LOGÍSTICA DA MARINHA, 19., 2019, Rio de Janeiro, RJ. Anais [...]. Rio de Janeiro: Centro de Análises de Sistemas Navais, 2019.

\section{INTRODUÇÃO}

A gestão de estoques tem o intuito de coordenar a demanda existente com a oferta de itens nas dimensões tempo e espaço. Assim, observando-se as peculiaridades de cada item, da operação e da demanda busca-se atingir os objetivos de custo e de nível de serviço especificados (WILLEMAIN; SMART; SCHWARZ, 2004). Nesse âmbito, Syntetos, Boylan e Croston (2005) afirmam que a tomada de decisão em gestão de estoques de peças de reposição é muito mais complexa.

Isso ocorre em função destes itens possuírem demanda intermitente, que é caracterizada pela presença de períodos com demanda zero em sua série histórica, com baixa variação do tamanho da demanda, quando ela ocorre (KOURENTZES, 2014). Peças de reposição de automóveis, aeronaves e navios, militares ou não, são alguns exemplos destes itens para Willemain, Smart e Schwarz (2004) e Syntetos e Boylan (2010).

Pequenas melhorias no sistema de uma organização em relação a esses itens podem ser traduzidas em grandes economias de recursos (SYNTETOS; BABAI; GARDNER, 2015). Assim, é notório o papel crucial que as previsões de demanda desempenham na gestão de estoques (WILLEMAIN; SMART; SCHWARZ, 2004), que devem ter seus modelos de previsão acompanhados e revisados constantemente (MAKRIDAKIS; WHEELWRIGHT; HYNDMAN, 1998) de modo a sempre garantir a melhor estimativa da demanda futura.

$\mathrm{Na}$ Marinha do Brasil (MB), conforme definem as Normas para a Execução do Abastecimento (BRASIL, 2009), as peças de reposição dos meios operativos e equipamentos são denominadas sobressalentes. Seu gerenciamento é realizado pelo Sistema de Abastecimento da Marinha (SAbM), que efetua a gestão de estoques e a previsão de demanda de sobressalentes através do Subsistema de Planejamento do Sistema de Informações Gerenciais do Abastecimento (SINGRA).

Nesse contexto, a partir da observação do emprego atual do SINGRA, definiu-se a justificativa, bem como demonstrou-se a relevância desta pesquisa em função do seguinte:

a) O Subsistema Planejamento não está sendo utilizado pelo Gestor da cadeia de sobressalentes do órgão de controle do SAbM para o apoio a tomada de decisões relacionadas com a previsão de demanda desta categoria de material (informação verbal) ${ }^{i}$;

b) De acordo com o Manual do SINGRA (BRASIL, 2007), os métodos disponíveis (Amortecimento Exponencial Simples, Média Móvel, Regressão Linear) são aplicados para todas as cadeias de suprimento (alimentação, fardamento, munição, sobressalentes, material de limpeza, escritório e saúde), sem considerar seus distintos comportamentos de demanda; e

c) Encontra-se em execução o projeto de modernização do SINGRA, com foco no Subsistema de Planejamento (informação verbal) ${ }^{\text {ii }}$. 
Diante do exposto, é possível levantar o seguinte questionamento: existem métodos quantitativos de previsão de demanda intermitente mais adequados aos itens da cadeia de sobressalentes do que os implementados atualmente no SINGRA? Por mais adequado, entende-se com o menor Erro Escalonado Médio Absoluto, cujo conceito operacional será doravante apresentado.

A pergunta acima será adotada como problema de estudo deste trabalho, sendo definido, a partir dela, o objetivo geral de identificar métodos de previsão de demanda intermitente que sejam mais adequados para itens de sobressalentes, a partir de uma comparação dos métodos em uso atualmente pelo SINGRA frente aos métodos que, segundo a literatura da área, possuem maior afinidade com as características da demanda destes itens.

Nesse sentido, os seguintes objetivos específicos foram definidos: (i) descrever a sistemática de previsão atualmente empregados pelo SINGRA na determinação corrente de necessidades de sobressalentes; (ii) identificar a principal característica da demanda dos sobressalentes para selecionar alguns métodos quantitativos, teoricamente alinhados à elas, com base na série histórica destes itens e no referencial teórico definido; e (iii) comparar os métodos implementados no SINGRA frente aos métodos selecionados na literatura e indicar, dentre os analisados, os métodos mais adequados para prever a demanda de sobressalentes.

Dessa forma, considerando que a literatura aponta para a predominância de demanda com característica intermitente quando se trata do peças de reposição, foram definidas as seguintes hipóteses a serem testadas pelos métodos quantitativos apresentados nesta pesquisa: (i) os itens de sobressalentes da $\mathrm{MB}$ apresentam, preponderantemente, característica de demanda intermitente; e (ii) métodos quantitativos de previsão de demanda sugeridos pela literatura da área para atender aos itens com característica de demanda intermitente são mais precisos que os disponíveis no SINGRA.

\section{REFERENCIAL TEÓRICO}

\subsection{PReVisão de DeMANDA}

Para Kourentzes (2014), são necessárias previsões de demanda precisas a fim de apoiar as decisões de manutenção e reposição de estoques das organizações. Sem estas previsões, as organizações não possuem as informações essenciais para auxiliar na realização de um planejamento adequado para futuros eventos inesperados, podendo, apenas, reagir a estes acontecimentos (PELLEGRINI; FOGLIATTO, 2001).

De forma geral, os métodos de previsão de demanda podem ser divididos em qualitativos e quantitativos. Métodos quantitativos utilizam modelos matemáticos e estatísticos para projetar a demanda a partir de séries temporais de dados históricos (MAKRIDAKIS; WHEELWRIGHT; HYNDMAN, 1998). Segundo Syntetos e Boylan (2010), na análise de série temporal as informações históricas são utilizadas para identificar padrões de demandas como o tamanho da demanda e o intervalo em que ela ocorre, a fim de replicá-las no futuro.

Diferentes métodos de previsão de demanda estão disponíveis para determinação do modelo mais adequado para cada situação (MAKRIDAKIS; WHEELWRIGHT; HYNDMAN, 1998). Entretanto, quando a demanda apresenta característica intermitente, é essencial a utilização de técnicas de previsão de demanda específicas para lidar com essa especificidade da demanda (PELLEGRINI; FOGLIATTO, 2001).

Dentre os métodos clássicos utilizados em estudos sobre demanda intermitente, destaca-se a utilização em conjunto de, ao menos, os métodos de Amortização Exponencial Simples (AES), Croston e Syntetos-Boylan Approximation (SBA) em trabalhos com diferentes abordagens e objetivos, como: Syntetos e Boylan (2005, 2010); Syntetos, Boylan e Croston (2005); Wallström e Segerstedt (2010); Babai, Ali e Nikolopoulos (2012); 
Kourentzes (2014); Syntetos, Babai e Gardner (2015); e Petropoulos, Kourentzes e Nikolopoulos (2016).

Essa frequente utilização demonstra a utilidade prática e acadêmica de tais métodos na previsão de demanda. Nesse âmbito, Wallström e Segerstedt (2010) afirmam que, a despeito da AES ainda ser um método difundido em diferentes sistemas de computadores e programas de previsão, a sua capacidade de previsão para itens com demanda intermitente é questionável, mesmo que em seu estudo esse método tenha apresentado bons resultados.

Nesse trato, Babai, Ali e Nikolopoulos (2012) comentam que métodos, como o AES e alguns modelos de médias móveis, tendem a gerar resultados com maior taxa de erro para os padrões de demanda intermitente. Da mesma forma, para Croston (1972), o amortecimento exponencial não é adequado nestes casos específicos, pois ele tende a superestimar a previsão.

Dessa forma, Croston (1972) propôs um método que se baseia explicitamente nas estimativas dos intervalos entre demandas e dos tamanhos de demanda, que atualmente é o método mais utilizado em muitas organizações, sendo implementado em diferentes sistemas Enterprise Resource Planning (ERP) e softwares de previsão de demanda (SYNTETOS; BOYLAN, 2010; PETROPOULOS; KOURENTZES; NIKOLOPOULOS, 2016).

Apesar de Croston (1972) afiançar que seu método não era enviesado, Syntetos e Boylan (2001) demonstraram que ele era positivamente tendencioso (havia excesso de previsão de demanda média) e, posteriormente, propuseram um estimador para corrigir o método, conhecido como Syntetos-Boylan Approximation (SBA), que apresenta melhores resultados na presença de demanda errática, irregular ou intermitente (SYNTETOS; BOYLAN, 2005).

Subsequentemente, Teunter e Sani (2009) e Wallström e Segerstedt (2010) mostraram que este método modificado ainda possui um viés quando o nível de intermitência da série histórica é baixo. Entretanto o método SBA é a única abordagem alternativa com substancial suporte empírico, demonstrando desempenho superior quando comparada ao método original (SYNTETOS; BABAI; GARDNER, 2015).

Recentemente, comprovou-se a eficácia de diferentes outros métodos perante os métodos clássicos, os quais têm sido estudados na literatura, como os baseados em: reamostragem (WILLEMAIN; SMART; SCHWARZ, 2004; TEUNTER; DUNCAN, 2009; SYNTETOS; BOYLAN; GARDNER, 2015), agregação temporal (PETROPOULOS; KOURENTZES; NIKOLOPOULOS, 2016), combinação de métodos e aprendizagem de máquina (MAKRIDAKIS; SPILIOTIS; ASSIMAKOPOULOS, 2018).

Syntetos, Boylan e Gardner (2015) afirmam que, apesar do resultado encontrado com a aplicação de reamostragem apresentar vantagens sobre os demais métodos, deve-se ter atenção à complexidade adicional exigida por esta metodologia. Dessa forma, concluíram que os métodos convencionais exigem menos poder computacional, o que é importante quando as demandas por números muito grandes de itens precisam ser previstas.

Por fim, salienta-se que Makridakis, Wheelwright e Hyndman (1998) afirmam que métodos mais complexos não necessariamente fornecem melhores previsões e que, segundo Kourentzes (2014), apesar destes novos métodos apresentarem bons resultados, ainda não têm sido amplamente utilizados. Esta condição sugere a continuidade da aplicação dos métodos quantitativos destacados inicialmente, que são apresentados na seção seguinte.

\subsection{Métodos Quantitativos de Previsão de Demanda}

No Quadro 1, apresentam-se as notações para os parâmetros que serão utilizadas nas equações dos métodos AES, Croston e SBA apresentadas a seguir, os quais servem de base para a consecução futura de parte do segundo objetivo específico determinado. 
Quadro 1 - Notação das equações de previsão de demanda

\begin{tabular}{|c|c|c|}
\hline Notação & Definição & Equação \\
\hline$\hat{y}_{\hat{t}}$ & Previsão calculada para o período $t$ & Todas \\
\hline$y_{t}$ & Demanda observada no período $t$ & Todas \\
\hline$\alpha$ & Parâmetro de amortização, entre 0 e 1 & Todas \\
\hline$z_{t}$ & Previsão do tamanho da demanda após o período $t$ & Croston e SBA \\
\hline $\mathrm{p}_{\mathrm{t}}$ & Previsão do intervalo de demanda após o período $t$ & Croston e SBA \\
\hline
\end{tabular}

Fonte: Elaborado pelo autor.

\subsubsection{Amortização Exponencial Simples (AES)}

O modelo de amortização exponencial consiste na atribuição de um peso para cada valor da série temporal, de forma que os valores mais recentes recebem ponderações maiores (PELLEGRINI \& FOGLIATTO, 2001). Segundo Makridakis, Wheelwright e Hyndman (1998), este modelo tem como principais vantagens a simplicidade e o baixo custo, fatores que levam estas técnicas a serem constantemente utilizadas.

Tradicionalmente, conforme descrevem Makridakis, Wheelwright e Hyndman (1998), utiliza-se o valor do primeiro período $\left(y_{1} y_{1}\right)$ como valor da primeira previsão $\left(\hat{\mathrm{y}}_{1} \hat{\mathrm{y}}_{1}\right)$ durante a fase de inicialização do método, com o parâmetro $(\alpha)$ igual a 0,15 ou 0,20 . Isso acontece em função da característica regressiva do método, sendo suas previsões estimadas a partir da equação:

$$
\hat{\mathrm{y}}_{t+1}=\hat{\mathrm{y}}_{t}+\alpha \times\left(y_{t}-\hat{\mathrm{y}}_{t}\right)
$$

\subsubsection{Modelo de Croston}

Croston (1972) desenvolveu um trabalho pioneiro no que tange à previsão de demanda para componentes ou eventos que apresentem comportamento intermitente. Ele sugeriu aplicar o AES separadamente sobre o tamanho da demanda e o intervalo entre demandas, de forma que o viés da aplicação de um único AES, a toda série temporal, fosse eliminado.

Segundo Eaves (2002), o método atualiza as estimativas quando há ocorrência de demanda, após aplicação separada do AES. Já no caso de não ocorrer demanda no espaço entre uma revisão do período e outra $(\mathrm{t})$, o método somente incrementa a contagem dos períodos, desde a última demanda, por meio da variável (q). Seu cálculo utiliza as equações:

$$
\begin{aligned}
z_{t}=\left\{\begin{array}{c}
y_{t}=0, \quad z_{t-1} \\
y_{t} \neq 0, z_{t-1}+\alpha \times\left(y_{t}-z_{t-1}\right)
\end{array}\right. & p_{t}=\left\{\begin{array}{c}
y_{t}=0, \quad p_{t-1} \\
y_{t} \neq 0, p_{t-1}+\alpha \times\left(q-p_{t-1}\right)
\end{array}\right. \\
\hat{y}_{t}=\frac{z_{t}}{p_{t}} & q=\left\{\begin{array}{c}
y_{t}=0, q+1 \\
y_{t} \neq 0,1
\end{array}\right.
\end{aligned}
$$

Por padrão, utiliza-se o mesmo parâmetro de amortização para as equações de $z_{t}$ e $p_{t}$ (KOURENTZES, 2014). Quanto ao seu valor, para Croston (1972), as melhores previsões ocorrem para $0,1 \leq \alpha \leq 0,3$. Syntetos e Boylan $(2005,2006)$ sugerem o uso de parâmetros entre 0,05 e 0,20 , enquanto Wallström e Segerstedt (2010) utilizaram constantes com 9 valores distintos entre 0,25 e 0,30. Nesse trato, Teunter e Duncan (2009) salientam que o efeito do uso diferentes parâmetros é pequeno quando analisada à diferença de desempenho que eles causam na comparação entre os vários métodos.

Por fim, cabe destacar que os dois parâmetros de previsão do método Croston são iniciados a partir de informações do primeiro ano da demanda, sendo o tamanho da demanda inicial calculado a partir da média das demandas observadas neste ano (EAVES, 2002). 


\subsubsection{Modelo de Syntetos-Boylan Approximation (SBA)}

Após Syntetos e Boylan (2001) demonstrarem que a formulação proposta por Croston (1972) tendia a superestimar o estoque, propuseram uma modificação em sua equação. A versão sugerida continua atualizando o tamanho e o intervalo da demanda, bem como utiliza o mesmo parâmetro de amortização da previsão do intervalo de demanda do método original (TEUNTER; DUNCAN, 2009), corrigindo-o conforme abaixo (SYNTETOS; BOYLAN, 2005):

$$
\hat{\mathrm{y}}_{t}=\left(1-\frac{\alpha}{2}\right) \times \frac{z_{t}}{p_{t}}
$$

\subsection{Modelo de Previsão de Demanda do Sistema de Abastecimento da Marinha}

Segundo o Manual do SINGRA (BRASIL, 2007), na atividade de determinação corrente de necessidades, o cálculo da previsão de demanda é realizado a partir da comparação do resultado dos seguintes métodos: Amortecimento Exponencial Simples, com o coeficiente de amortecimento de 0,4; Média Móvel, para cinco períodos; e Regressão Linear, a partir de cinco períodos. No Quadro 2, apresentam-se os dois últimos métodos supracitados.

Quadro 2 - Métodos da Média Móvel e Regressão Linear

\begin{tabular}{|c|c|c|}
\hline Método & Equação & \multicolumn{1}{c|}{ Descrição } \\
\hline $\begin{array}{c}\text { Média } \\
\text { Móvel }\end{array}$ & $\hat{y}_{t+1}=\frac{1}{n} \times \sum_{t=1}^{n} y_{t}$ & $\begin{array}{l}\text { A média móvel considera como previsão para o período futuro a média das } \\
\text { observações passadas mais recentes. Ou seja, consiste no cálculo da média } \\
\text { dos valores mais recentes, de forma que a cada período, o valor mais antigo } \\
\text { é substituído por um mais recente, assim modificando a média. }\end{array}$ \\
\hline $\begin{array}{c}\text { Regressã } \\
\text { o Linear }\end{array}$ & $y=a+b \times x$ & $\begin{array}{l}\text { A regressão é uma estimativa da relação entre variáveis dependentes (y) e } \\
\text { independentes (x). Com base em sua equação, pode-se determinar, a partir } \\
\text { das informações dos pontos amostrais, a reta que melhor se ajusta a eles } \\
\text { pelo cálculo dos parâmetros } a \text { e } b .\end{array}$ \\
\hline
\end{tabular}

Fonte: Adaptado pelo autor com base em Makridakis, Wheelwright e Hyndman (1998).

Além destes cálculos, o sistema realiza o cálculo do Desvio Absoluto Médio (DAM) dos cinco últimos períodos chegando ao método que apresenta o menor desvio em relação à demanda real. Em seguida, sugere a previsão de demanda, calculada com este mesmo método para o período seguinte. Os períodos em questão correspondem aos valores de demanda agregados trimestralmente e que se utilizam os mesmos métodos para qualquer característica de demanda. Neste trabalho, esta metodologia será identificada por "Método SINGRA".

\subsection{Medidas da ACURácia da Previsão}

Após o cálculo da previsão de demanda, é necessário avaliar o desempenho de cada método, o que pode ser feito por meio da mensuração da diferença entre o valor estimado e a demanda observada no período (MAKRIDAKIS; WHEELWRIGHT; HYNDMAN, 1998).

Dentre as diversas medidas de erros abordadas na literatura, Wallström e Segerstedt, (2010) e Kourentzes (2014) citam o Desvio Absoluto Médio (DAM) e o Erro Quadrático Médio (EQM) como as mais comuns, que demonstram que uma previsão é mais precisa quanto menores forem os valores calculados por essas medidas de acurácia.

Apesar destas medidas terem apresentado alguns bons resultados na mensuração das previsões para demanda intermitente, como em Boylan, Syntetos e Karakostas (2008), Kourentzes (2014) alerta que o DAM e EQM tendem a polarizar as previsões em favor da previsão de demanda zero e não são uma boa referência para as decisões de estoque associadas às previsões. Além disso, elas não levam em consideração as diferenças de escala quando utilizadas em muitas séries temporais (SYNTETOS; BOYLAN, 2005).

Para essa situação, Syntetos e Boylan (2005) sugerem que quando mais de dois métodos de previsão estão envolvidos no exercício de comparação, é útil relatar a proporção 
de vezes que um método tem um desempenho melhor do que todos os outros métodos. Nesse sentido, sugerem apresentar o Melhor Percentual (MP), que é capaz de apontar qual dos métodos é mais vezes melhor que os demais.

Apesar de ser um bom estimador da quantidade de vezes que um método é melhor que outros métodos, o MP não oferece nenhuma informação da qualidade da melhoria oferecida pelo método (SYNTETOS; BOYLAN, 2006), essa condição sugere a utilização de outro estimador capaz de medir a qualidade desta superioridade de acurácia.

Neste contexto, Hyndman e Koehler (2006) conduziram um estudo que abordou medidas relativas, dependentes da escala, baseadas em erros percentuais e relativos, com foco na demanda intermitente. Analisando os pontos fortes e fracos de cada métrica, sugeriram a utilização do Erro Escalonado Médio Absoluto (Mean of Absolute Scaled Error, MASE), uma medida de erro escalonado que é adequada às condições em que os demais métodos apresentam distorções e tem sido utilizada em estudos recentes, como por Makridakis, Spiliotis e Assimakopoulos (2018).

Se o valor do MASE for menor do que um, conclui-se que as previsões calculadas pelo método proposto, em média, são menos errôneas do que as previsões calculadas pelo Método Ingênuo, aquele que apenas repete a previsão real observada em um período como previsão para o período subsequente.

Ademais, Hyndman e Koehler (2006) afirmam que a principal vantagem desta medida é a adequação ao erro de séries temporais com características de demanda intermitente, servindo igualmente para comparação da acurácia das previsões estimadas a partir de métodos alternativos e para diferentes séries de dados. No Quadro 3, apresentam-se as equações das medidas DAM e MASE de acurácia.

Quadro 3 - Medidas de Acurácia

\begin{tabular}{|c|c|l|}
\hline Método & Equação & \multicolumn{1}{c|}{ Descrição } \\
\hline DAM & $\frac{1}{n} \times \sum_{t=1}^{n}\left|y_{t}-\hat{y}_{t}\right|$ & $\begin{array}{l}\text { Seu cálculo consiste na média dos erros absolutos entre as } \\
\text { demandas observadas }\left(y_{t} y_{t}\right) \text { e as previsões calculadas }\left(\hat{\mathrm{y}}_{t} \hat{\mathrm{y}}_{\mathrm{t}}\right), \\
\text { ambas para o período t. }\end{array}$ \\
\hline MASE & Média $\frac{y_{t}-\hat{\mathrm{y}}_{t}}{\frac{1}{n-1} \sum_{t=2}^{n}\left|y_{t}-y_{t-1}\right| \rrbracket}$ & $\begin{array}{l}\text { Este cálculo é resultado da divisão do erro da previsão }( \\
\left.\left.y_{t}-\hat{\mathrm{y}}_{t}\right) y_{t}-\hat{\mathrm{y}}_{t}\right), \text { medido por uma determinada métrica, pela } \\
\text { média dos erros, na amostra, do Método Ingênuo. }\end{array}$ \\
\hline
\end{tabular}

Fonte: Adaptado pelo autor com base em Hyndman e Koehler (2006).

Cabe ainda destacar que, devido à natureza dos dados intermitentes, o uso de medidas de erros convencionais tem sido questionado (TEUNTER; DUNCAN; 2009). Assim, outras medidas têm sido sugeridas, como as baseadas em nível de serviço ao cliente (BOYLAN, SYNTETOS, KARAKOSTAS, 2008), as que avaliam as consequências dos erros da previsão (WALLSTRÖM; SEGERSTEDT, 2010) e funções do custo (KOURENTZES, 2014).

\subsection{ClassificaÇão da DEMANDA}

As regras de categorização de demanda apoiam a seleção dos métodos de previsão e de controle de estoque que devem ser usados para cada tipo de item diferente. Consequentemente, essas regras utilizadas têm implicações significativas em termos de níveis de estoque e satisfação do cliente (BOYLAN; SYNTETOS; KARAKOSTAS, 2008).

Neste sentido, Syntetos, Boylan e Croston (2005) sugerem uma classificação por meio de parâmetros e valores de corte para tais medidas (Figura 1). Os parâmetros utilizados são (i) o quadrado do coeficiente de variação $\left(\mathrm{CV}^{2}\right)$, calculado por meio do quadrado da divisão do desvio-padrão da demanda pela demanda média, ambas para valores de demanda diferentes de zero; e (ii) o intervalo médio entre demandas (ADI), que corresponde ao número médio de períodos de tempo entre duas demandas sucessivas. 
Figura 1 - Categorias de demanda de peças de reposição

Fonte: Adaptado de Syntetos, Boylan e Croston (2005).

A partir da Figura 1, quatro categorias de demanda podem ser definidas: (i) Regular, corresponde às peças de alto giro e com baixa variação de quantidade; (ii) Intermitente, relativa às peças com quantidade de períodos sem demanda (baixo giro) e com baixa variação de quantidade; (iii) Errática, as que possuem alto giro e alta variabilidade da quantidade; e (iv) Irregular, caracterizado por possuir muitos períodos sem demanda (baixo giro) e alta variabilidade da quantidade (SYNTETOS; BOYLAN; CROSTON, 2005).

Essa caracterização detalhada da demąıda ${ }_{1,32}$ peças de reposição foi utilizada por Boylan, Syntetos e Karakostas (2008) e Wallström e Segerstedt (2010). Entretanto, Syntetos (2007) alerta que tais valores de corte nã tendo sido calculados sob a premissa de identificar quando um nétodo de previsão supera ou ro. Ademais, para estimação

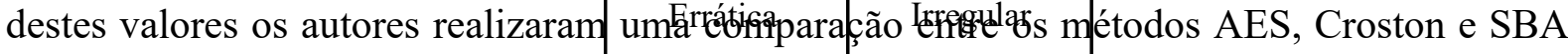
com base na utilização EQM para 3.000 peças de reposição.

Destarte, esses valoxes pgodeqn variar de acprdo com a análise feita, principalmente se forem utilizados outros métodos de preyisão (\$YNTFTfQS 2007). Neste contexto, para utilizar corretamente esta classificação, faz-se necessário que se atente às premissas que permearam o cálculo dos valores de corte, de manqira a não invalidar o seu uso.

\section{METODOLOGIA DE PESQUISA}

\subsection{TIPO De Pesquisa}

Segundo Prodanov e Freitas (2013), este estudo com abordagem quantitativa é classificado como uma pesquisa aplicada, segundo sua natureza, e exploratória, em relação aos seus objetivos. Quanto aos procedimentos, a fim de atingir os objetivos específicos estabelecidos, realizou-se:

a) Uma pesquisa documental, valendo-se de materiais que não receberam ainda um tratamento analítico, fontes de primeira mão (GIL, 2008), com fulcro em documentos normativos da MB que tratam sobre o tema;

b) Uma pesquisa bibliográfica, de acordo com Prodanov e Freitas (2013), baseada em livros, periódicos e artigos científicos, acerca dos temas: (i) peças de reposição; (ii) características de sua demanda; (iii) seus métodos de previsão de demanda; e (iv) métricas de medição da acurácia das previsões; e

c) Um estudo de caso aplicado no SAbM, nas condições contextuais tratadas por Yin (2001) e com o propósito de "descrever a situação do contexto em que está sendo feita determinada investigação" (PRODANOV; FREITAS, 2013, p. 46), em que foram comparados os métodos de previsão de demanda utilizados pela $\mathrm{MB}$ e os evidenciados na pesquisa bibliográfica com o fito de verificar quais apresentavam o menor erro. 


\subsection{Coleta e Tratamento de Dados}

Para coleta de dados, no contexto definido por Prodanov e Freitas (2013), realizou-se uma pesquisa direta intensiva por meio da técnica de entrevistas não estruturadas com o gestor da cadeia de sobressalentes do SAbM e o encarregado do Apoio Logístico Integrado do SAbM para evidenciar dados primários sobre como, na prática, os recursos de previsão de demanda apontados pela pesquisa documental são utilizados.

Além destes, também foram coletados os dados da série histórica que serviu como base para comparação entre métodos de previsão de demanda, objetivo deste trabalho. Essa série histórica foi fornecida pela Diretoria de Abastecimento da Marinha, órgão responsável pelo SAbM e administrador do SINGRA, e corresponde às informações dos registros mensais de demanda de 29.958 itens sobressalentes de máquinas e motores referentes ao período compreendido entre janeiro de 2011 e junho de 2018.

Estes dados representam o consumo de sobressalentes para manutenções (preventivas e corretivas) de diferentes Organizações Militares que utilizam esses itens. Já na fase de tratamento destes dados, seguiu-se a seguinte metodologia:

a) A série histórica foi agregada em trimestres, resultando em uma série de 30 trimestres, a fim de se estabelecer a mesma base temporal utilizada pelo SINGRA e permitir a posterior comparação entre métodos de previsão. Salienta-se que este procedimento não prejudica a previsão, podendo inclusive contribuir para a performance da previsão por reduzir a intermitência da série (PETROPOULOS; KOURENTZES; NIKOLOPOULOS, 2016);

b) Como utilizado por Pellegrini e Fogliatto (2001), empregou-se a regra de Pareto (Classificação $\mathrm{ABC}$ ), que determina a importância do produto, relacionando a demanda e o seu faturamento, para selecionar os itens de maior importância financeira, elegendo-se 1.983 itens (Classe A), que representam $80 \%$ do volume monetário movimentado no período;

c) Conforme sugerem Petropoulos, Kourentzes e Nikolopoulos (2016), o total de 30 trimestres foi divido em dois conjuntos: o primeiro de inicialização (26 trimestres), onde foram estabelecidos os requisitos iniciais para rodagem dos métodos de previsão, como descrevem Makridakis, Wheelwright e Hyndman (1998), para o método AES, e Eaves (2002), para o método Croston; e o segundo de desempenho (4 trimestres), para a efetiva comparação e mensuração da acurácia das previsões;

d) De maneira similar à metodologia de seleção de itens hábeis a serem utilizados na previsão de demanda aplicada por Boylan, Syntetos e Karakostas (2008), estabeleceu-se o critério de que a série histórica de cada item possua, no mínimo, dois registros de demanda não nula nos últimos cinco períodos (definido a partir da amostra temporal da média móvel do Método SINGRA), excluindo-se o período de desempenho. Essa restrição garante que todos os valores iniciais possam ser estimados até o final do bloco de inicialização e reduziu o número de sobressalentes em análise para 402 itens;

e) Os itens de sobressalentes foram segregados de acordo com a categorização proposta por Syntetos, Boylan e Croston (2005), para verificação de qual era a principal característica da demanda presente nos 402 itens de sobressalentes;

f) Selecionaram-se os métodos de previsão de demanda mais adequados a categoria de demanda que se mostrou predominante em relação às demais no processo de categorização;

g) O processamento de dados e o cálculo das previsões foram realizados por meio do Software Microsoft Office Excel® para previsões de quatro períodos a frente, conforme evidenciado no estudo de caso, e utilizando parâmetros de amortização $(\alpha)$ fixados em valores de $0,05,0,10,0,15,0,20,0,25$ e 0,30 , escolhidos com base nos estudos de Croston (1972) e Syntetos e Boylan $(2005,2006)$, autores de dois dos métodos selecionados; e 
h) Analisaram-se os resultados das comparações efetuadas no período de desempenho tendo o MASE como medida de acurácia, somente para os itens que correspondem a característica de demanda predominante. Em seguida, o resultado de quantas vezes cada método foi mais preciso que os outros foi demonstrado pelo uso do Melhor Percentual (MP).

\section{ESTUDO DE CASO}

\subsection{Sistema de Abastecimento da Marinha (SAbM)}

Conforme descrito nas Normas para a Execução do Abastecimento (SGM-201), o "Abastecimento é um conjunto de atividades que tem o propósito de prever e prover, para as Forças e demais $\mathrm{OM}$ da $\mathrm{MB}$, o material necessário a mantê-las em condições de plena eficiência" (BRASIL, 2009, p. 1-1). Ou seja, as atividades do abastecimento objetivam promover, manter e controlar o adequado provimento do material à MB.

Para seu funcionamento, o SAbM dispõe do Sistema de Informações Gerenciais do Abastecimento (SINGRA), ERP utilizado pelo SAbM, para realização de todas as atividades que permitem o cumprimento de sua missão. Dentre os subsistemas do SINGRA, destaca-se o de planejamento, onde é possível realizar a gerência dos estoques do SAbM através da análise de demanda, da verificação dos níveis de estoque e da emissão de pedidos de compra, atividade conhecida como Controle de Inventário, conforme SGM-201 (BRASIL, 2009).

\subsection{CONTROLE DE INVENTÁRIO DO SABM}

Sempre que um novo meio operativo ou equipamento é incorporado à $M B$, são estabelecidas as quantidades de itens de sobressalentes que devem ser mantidos a bordo dele e nos depósitos, para a reposição destes e para manutenção preventiva e corretiva, de forma que sempre se garanta que um meio tenha as peças de reposição necessárias quando preciso.

De acordo com a SGM-201 (BRASIL, 2009), estas quantidades são conhecidas como dotações iniciais de bordo e de base, que são calculadas inicialmente por meio da atividade chamada de Determinação Técnica de Necessidades. Em sequência, o SAbM corrige essas estimativas iniciais, estabelecendo as reais necessidades de material para um determinado período a partir da observação de índices relativos ao acompanhamento da demanda real, dentre eles a frequência de pedidos e suas respectivas quantidades, atendidas ou em dívida.

Conforme descreve o Manual do SINGRA (BRASIL, 2007), essa atividade é denominada Determinação Corrente de Necessidades, sendo esta fixação de necessidades feita em termos de níveis de estoque, que seguem os padrões variados da evolução da demanda dos sobressalentes. Dessa forma, a partir da comparação entre as necessidades e as disponibilidades, apresentadas pelo controle de estoque, é realizado o Controle de Inventário.

Nessa sistemática, cabe salientar que o modelo para a determinação de necessidades e o controle de inventário se pauta na realização de cálculos da previsão da demanda para o estabelecimento dos níveis de estoque com base no lead time de entrega de cada item. Assim, o sistema apresenta uma sugestão de encomenda, que deverá ser adquirida em determinado momento para a manutenção do nível de serviço previamente estabelecido (BRASIL, 2007).

Entretanto, a informação da previsão de demanda do Subsistema Planejamento do SINGRA não tem sido utilizada em função de ser gerada a partir de métodos de previsão de demanda simples, que não levam em consideração o tipo de item em análise. Assim, por serem pouco adequados à característica dos sobressalentes, geram grandes erros. Além disso, a previsão só é fornecida para um período a frente (três meses), enquanto, que em média, os sobressalentes demoram um ano para serem recebidos pelo SAbM (informação verbal) ${ }^{1}$.

Nesse sentido, atualmente estão sendo conduzidos estudos com o objetivo de atualizar o sistema como um todo, como foco principal no Subsistema Planejamento, onde está inserida a funcionalidade da previsão de demanda. Nesses estudos, a fim de aprimorar o 
funcionamento do SAbM, estão buscando reavaliar as teorias que dão sustentação às regras de negócios deste Subsistema, dentre elas os métodos de previsão demanda de todas as categorias de material, incluindo os sobressalentes (informação verbal) ${ }^{2}$.

\section{ANÁLISE EMPÍRICA}

Em princípio, com base na pesquisa documental, cumpriu-se o primeiro objetivo específico ao descrever a sistemática de previsão atualmente empregada pelo SINGRA na determinação corrente de necessidades de sobressalentes, conforme descrito no item 2.1 deste trabalho e denominada "Método SINGRA". Destarte, nesta fase da pesquisa, com o objetivo de atender aos demais objetivos específicos, serão realizadas a:

a) Categorização da demanda dos itens de sobressalentes, com objetivo de testar a primeira hipótese, de que os itens de sobressalentes da MB apresentam, preponderantemente, característica de demanda intermitente;

b) Seleção dos métodos de previsão de demanda que tenham maior afinidade com a característica de demanda preponderante, conforme descrito na literatura da área; e

c) Comparação dos métodos selecionados frente o Método SINGRA, definida conforme descrito na metodologia, para testar a segunda hipótese, de que métodos quantitativos de previsão de demanda sugeridos pela literatura para atender aos itens com característica de demanda intermitente produzem ganhos médios superiores aos métodos em uso no SINGRA.

\subsection{Categorização da Demanda e Seleção dos Métodos de Previsão}

Para verificar a primeira hipótese estabelecida, foram calculados os valores do intervalo médio entre demandas (ADI) e quadrado do coeficiente de variação $\left(\mathrm{CV}^{2}\right)$ para cada um dos itens. Depois, utilizando-se os valores de corte apresentados anteriormente os itens foram segregados nas categorias de demanda propostas por Syntetos, Boylan e Croston (2005).

Assim, chegou-se à conclusão de que 276 itens têm característica intermitente $(68,7 \%)$, 115 itens apresentam característica irregular $(28,6 \%)$ e 11 itens possuem característica de demanda do tipo errática (2,7\%). Isto posto, pode-se concluir que, efetivamente, os itens de sobressalentes possuem a característica preponderantemente intermitente em sua demanda, estando, dessa forma, de acordo com o previsto na literatura para as peças de reposição e confirmando a primeira hipótese estabelecida para esta pesquisa.

A partir da categoria de demanda intermitente identificada foram selecionados os seguintes métodos de previsão: Amortecimento Exponencial Simples (AES), Croston e Syntetos-Boylan Approximation (SBA). Essa seleção baseou-se em dois princípios. O primeiro faz relação com a quantidade de estudos que utilizaram, pelo menos, estes três métodos em comparação com outros, que foi demonstrado no referencial teórico abordado.

O segundo diz respeito a limitação citada por Syntetos (2007) quanto à utilização da metodologia proposta para categorização, que é diretamente impactada quando se utilizam métodos de previsão diferentes dos empregados para determinar os valores de corte sugeridos.

\subsection{Comparação entre Métodos e Medição da ACURÁcia}

Nesta fase da análise empírica, os métodos selecionados serão comparados com o Método SINGRA para previsão de 1 ano (quatro períodos) a frente, período de previsão que foi definido tendo como referência o prazo médio de entrega identificado na entrevista com o Gestor da Cadeia de Sobressalentes do SAbM (informação verbal) ${ }^{1}$.

Partindo desse ponto, por meio da utilização de planilhas eletrônicas, os métodos AES e Croston foram iniciados da mesma forma com o objetivo de homogeneizar ao máximo às previsões realizadas. Nesse sentido, seguindo Makridakis, Wheelwright e Hyndman (1998) e 
Eaves (2002), calculou-se a média aritmética dos quatro primeiros períodos (1 ano), adotando seu resultado como a previsão para o quarto período. Destarte, as previsões com base nas equações dos métodos citados foram iniciadas no quinto período.

Croston (1972) alerta para a importância de controlar desvios de previsão em função da frequente atualização do método, que pode ter como efeito atrasos nas respostas reais dos parâmetros. Assim, sugere a medição por meio de quatro indicadores, os quais foram testados de maneira a verificar-se que não houve nenhuma limitação ao uso do método.

Quanto ao método SBA, como ele corresponde a uma correção do método original de Croston (1972), aplicou-se apenas a correção sugerida por Syntetos e Boylan (2005) ao método original já inicializado. Dessa forma, foram realizadas previsões para cada período durante o período de inicialização (dentro da amostra), que corresponde aos 26 primeiros trimestres e, a começar do vigésimo sétimo trimestre até o trigésimo, estimaram-se as previsões fora da amostra, no bloco de desempenho.

Conforme descrito na metodologia, estas previsões foram realizadas com base em parâmetros de amortização com valores de $0,05,0,10,0,15,0,20,0,25$ e 0,30 , escolhidos com base nos estudos de Croston (1972), que utilizou valores entre 0,1 e 0,3 e Syntetos e Boylan (2005, 2006), que utilizaram valores entre 0,05 e 0,20 .

Cabe ressaltar que a metodologia de previsão de demanda do SINGRA foi reproduzida em planilhas eletrônicas com objetivo de facilitar o estudo e não criar uma dependência do sistema. Assim, as informações, das previsões por esse método, foram calculadas por meio destas planilhas, previamente certificadas pelos responsáveis pela gerência da cadeia de sobressalentes do SAbM, conforme o Manual do SINGRA (BRASIL, 2007).

Após calculadas as previsões de todos métodos para um item, foram medidos os erros (diferença entre o valor real observado e o valor estimado) de cada estimativa por meio do MASE. Posteriormente, essas medidas foram comparadas para evidenciar o método que apresentou o menor MASE na comparação, ou seja, o método que melhor se aplica ao item analisado. Esse procedimento foi aplicado para todos os 276 itens de demanda intermitente.

Preferiu-se utilizar o MASE como medida de acurácia em função dele ser claramente independente da escala das séries temporais (HYNDMAN; KOEHLER, 2006). Nesse sentido, pode ser utilizado para estabelecer relações entre elas, mesmo quando há registro de demanda intermitente na base de dados em análise.

A partir do desenvolvimento narrado acima, são apresentados na Tabela 1 o ranking dos métodos para os itens de sobressalentes com característica de demanda errática. O escalonamento foi elaborado por meio da medição do percentual de quantas vezes cada um dos métodos foi o melhor do que todos os utilizados na comparação com base no MASE, sendo representado pelo Melhor Percentual (SYNTETOS; BOYLAN, 2005).

Tabela 1 - Melhor Percentual (MASE) para previsão de quatro períodos a frente

\begin{tabular}{ccccccc}
\hline Rankin & \multicolumn{5}{c}{ Parâmetros de Amortização } \\
\cline { 2 - 6 } $\mathbf{g}$ & $\mathbf{0 , 0 5}$ & $\mathbf{0 , 1 0}$ & $\mathbf{0 , 1 5}$ & $\mathbf{0 , 2 0}$ & $\mathbf{0 , 2 5}$ & $\mathbf{0 , 3 0}$ \\
\hline $1^{\circ}$ & AES (49\%) & SBA (43\%) & SBA (54\%) & SBA (56\%) & SBA (59\%) & SBA (60\%) \\
& SINGRA & & & & SINGRA \\
$2^{\mathbf{o}}$ & $(24 \%)$ & SINGRA (25\%) & SINGRA (23\%) & SINGRA (23\%) & SINGRA (20\%) & $(18 \%)$ \\
$3^{\circ}$ & SBA (15\%) & AES (21\%) & AES (13\%) & AES (13\%) & AES (13\%) & AES (14\%) \\
$4^{\circ}$ & Croston (12\%) & Croston (11\%) & Croston (10\%) & Croston (8\%) & Croston (8\%) & Croston (8\%) \\
\hline
\end{tabular}

Fonte: Elaborado pelo autor.

Por meio desta Tabela, é possível identificar que, por exemplo, o método AES, quando utilizado com o parâmetro de amortização de 0,05 , foi em, aproximadamente, $49 \%$ dos casos, o método mais adequado aos itens de demanda de intermitente. 
Com base nessa interpretação, é possível visualizar que o método SBA mostrou-se mais vezes superior aos demais na previsão para quatro períodos a frente, seguido pelos métodos SINGRA, AES e Croston. Esta configuração de ranking foi unânime para os parâmetros de amortização com valores de 0,10 a 0,30 , tendo como exceção apenas a classificação estabelecida para o menor parâmetro, quando se inverteram as posições entre os métodos AES, que assumiu o primeiro lugar, e o método SBA, que caiu para o terceiro lugar.

Nesse trato, tem-se uma disposição geral clara da frequência com que cada método foi o mais adequado para cada item de sobressalente analisado, principalmente para os parâmetros maiores que 0,05 , o que leva a uma conclusão quanto às constantes de amortização utilizadas.

Pode-se perceber que apesar destes parâmetros apresentarem algum efeito no desempenho dos métodos (mudança do percentual de cada um), este efeito é pequeno em comparação à diferença de desempenho entre os vários métodos (o método que efetivamente é mais adequado à característica da demanda permaneceu vencedor independentemente do valor da constante), conforme também concluíram Teunter e Duncan (2009).

Analisando cada método de forma individual, o fato do método SBA mostrar-se mais preciso que os métodos Croston, AES e os métodos incutidos no SINGRA, quando aplicado com parâmetros entre 0,1 e 0,3 , leva a dois achados. O primeiro é a coerência com o esquema de classificação proposto por Syntetos, Boylan e Croston (2005), que sugere a utilização do método SBA para itens com demanda intermitente.

Já o segundo, ilustra a relevância de determinar as áreas em que cada método tem melhor performance de modo a otimizar o resultado da comparação, como sugere Syntetos (2007). Todavia, cabe salientar que o SBA não obteve bons resultados quando aplicado com o menor parâmetro selecionado. Este fato pode ser resultado da utilização de diferentes métricas de precisão, tendo em vista que Syntetos, Boylan e Croston (2005) utilizaram o EQM, sendo necessário realizar pesquisas futuras para identificar o motivo de deste achado.

Quanto ao Método SINGRA, apesar de ter ocupado a segunda posição para todos os parâmetros, foi o melhor para, no máximo, 25\% dos itens em análise. Esse fato demonstra a defasagem da sistemática empregada no SINGRA e sugere que a ausência de uma estruturação de métodos específica para cada cadeia de suprimento, bem como a não utilização de nenhum método desenvolvido especificamente para prever a demanda de séries com intermitência, prejudica a acurácia da previsão deste método.

No caso do método AES, apesar de não ter oferecido boas previsões de forma geral, destaca-se seu melhor resultado com o mesmo parâmetro que tornou o método mais preciso no estudo de Wallström e Segerstedt (2010), mas que diverge de outros valores sugeridos pela literatura, entre 0,15 e 0,2 (SYNTETOS; BOYLAN; 2005). Contudo, tendo em vista que estes dois estudos mediram os erros de previsão a partir do EQM e que as características das amostras são distintas, não é possível depreender maiores conclusões.

Por fim, o método Croston apresentou os piores resultados para todos os parâmetros de amortização, resultado que está coerente com as conclusões de Syntetos e Boylan (2006) e vai de encontro as proposições do próprio autor em dois fatores. $\mathrm{O}$ primeiro diz respeito à expectativa de que este método superasse os métodos AES e SINGRA, pelo menos para previsões maiores que 0,05 . O segundo é indicado pelo fato de o melhor resultado do método ter sido encontrado quando a constante era 0,05, valor não sugerido por Croston (1972).

Em que pese a necessidade de futuros estudos para uma análise aprofundada da variabilidade do resultado deste método, cabe ressaltar um ponto positivo de seus resultados. Dentre os métodos de previsão de demanda comparados, o método Croston apresentou a menor sensibilidade em relação a mudança de parâmetros, variando entre $8 \%$ a $12 \%$. Enquanto, por exemplo, o SBA variou de $43 \%$ a $60 \%$. 
Com objetivo de resumir os melhores resultados de cada método na comparação, é apresentado na Tabela 2 o ranking dos métodos de acordo com os maiores Melhores Percentuais de cada um deles, juntamente com os parâmetros que otimizaram estes resultados.

Tabela 2 - Melhor Percentual (MASE) de cada método na análise

\begin{tabular}{ccc}
\hline Ranking & Método & Parâmetro de Amortização \\
\hline $1^{\circ}$ Lugar & SBA $(60 \%)$ & 0,30 \\
$2^{\circ}$ Lugar & AES $(49 \%)$ & 0,05 \\
$3^{\circ}$ Lugar & SINGRA $(25 \%)$ &.. \\
$4^{\circ}$ Lugar & Croston $(12 \%)$ & 0,05 \\
\hline
\end{tabular}

Fonte: Elaborado pelo autor.

Em princípio, cabe destacar que, apesar do maior valor do Melhor Percentual do Método SINGRA ter sido apresentado quando o parâmetro de amortização foi 0,10 , não faz sentido apresentar este número, já que as previsões calculadas por esse método não levaram em consideração os valores dos parâmetros utilizados na análise.

Assim, finalmente, pode-se visualizar que na comparação entre os métodos de previsão de demanda intermitente selecionados a partir do referencial teórico estabelecido e os métodos implementados no SINGRA, apenas os métodos SBA e AES demonstram ser frequentemente melhores estimadores da demanda para os itens analisados. Resultados que são maximizados, quanto maiores os valores do parâmetro de amortização, para o método SBA e, quando o parâmetro utilizado é de 0,05 , para o método AES.

Esses fatos resultam na negação da segunda hipótese elaborada anteriormente, já que o método Croston não acompanhou o mesmo resultado dos outros dois métodos selecionados a partir da literatura, demonstrando superar o Método SINGRA somente em poucos casos.

No contexto desta análise, o Método SINGRA já prevê a utilização do método AES em sua sistemática, mas com o valor da constante de amortização fixado em 0,4 . Destarte, em função dos resultados evidenciados neste trabalho, sugere-se que a simples modificação deste parâmetro no SINGRA já pode fazer com que os resultados de previsão sejam mais precisos.

Por fim, frisa-se que a abordagem apresentada, Melhor Percentual, é útil apenas para demonstrar quantas vezes um método teve maior acurácia que os demais. Todavia, ela não fornece a informação da qualidade do ajuste de cada método em relação a referência (Método SINGRA), sendo necessária a utilização de outros métricas de mensuração de erro para essa finalidade (SYNTETOS; BOYLAN, 2005). Destarte, pela limitação do espaço disponível, esta mensuração da qualidade da previsão é colocada como sugestão de estudos futuros.

\section{CONCLUSÃO}

Petropoulos, Kourentzes e Nikolopoulos (2016) afirmam que as organizações muitas vezes detêm mais estoque do que o necessário para contabilizar as previsões de demanda ruins de itens com esse tipo de demanda. Para que erros grosseiros de previsão de demanda intermitente sejam evitados é preciso que estas previsões sejam feitas de forma mais precisa e menos variável, de forma que uma quantidade excessiva de itens, que representam um alto custo de inventário, não seja mantida em estoque (SYNTETOS; BOYLAN, 2010).

Nesse contexto, este trabalho teve o propósito de identificar métodos de previsão de demanda intermitente que fossem mais adequados para itens de sobressalentes de máquinas e motores da $\mathrm{MB}$, a partir de uma comparação dos métodos em uso atualmente pelo SINGRA frente aos métodos que, segundo a literatura da de previsão de demanda, possuem são mais adequados às características da demanda dos itens de sobressalentes.

Com base na pesquisa documental foi possível descrever, dentro da atividade de determinação corrente de necessidades, quais métodos de previsão de demanda são utilizados pelo SINGRA no cálculo de sua sugestão de previsão de demanda, informação que foi 
complementada pelo estudo de caso para verificar em qual contexto essa previsão estava inserida e como era utilizada no Sistema de Abastecimento da Marinha.

Já por meio da pesquisa bibliográfica, estabeleceram-se: os métodos de previsão de demanda intermitente mais adequados às peças de reposição; as medidas de acurácia necessárias para medir os erros das previsões e indicar os mais precisos; e os critérios de categorização de peças de reposição em quatro classes distintas, dentre elas a intermitente.

Assim, identificou-se que os itens de sobressalentes da MB possuem característica de demanda, predominantemente, intermitente (276 itens do total de 402 analisados), o que confirmou a primeira hipótese estabelecida para a pesquisa. Em sequência, foram selecionados os métodos Amortecimento Exponencial Simples (AES), Croston e SyntetosBoylan Approximation (SBA) para comparação frente o método SINGRA.

As previsões foram calculadas a partir de uma série de dados de 30 trimestres de 276 itens de sobressalentes como demanda intermitente para quatro períodos a frente (1 ano). Os erros de cada método foram mensurados a partir do MASE e, em função da realização da comparação entre diferentes métodos, utilizou-se o Melhor Percentual para apresentar a informação da quantidade de vezes que cada método foi melhor dentre todos os comparados.

Como resultado, observou-se que dentre os métodos selecionados na literatura, somente os métodos SBA (com parâmetros de amortização entre 0,10 e 0,30) e AES (com parâmetro de amortização igual a 0,05) demonstram ser frequentemente mais adequados do que o método SINGRA para previsão de demanda de sobressalentes, enquanto que o método Croston não apresentou bons resultados na comparação. Esta conclusão indica a negação da segunda hipótese, já que o método Croston não superou previsão sugerida pela sistemática do SINGRA.

Nesse sentido, afirma-se que existem métodos quantitativos de previsão de demanda intermitente mais adequados aos itens da cadeia de sobressalentes do que os implementados atualmente no SINGRA, dentre eles os métodos SBA e AES, quando utilizados nas condições evidenciadas nesta pesquisa.

Cabe registrar que uma limitação desta pesquisa se refere a base de dados, já que ela não contém informações da demanda não atendida, o que ocorre quando uma Organização Militar solicita um item ao SAbM e ele não existe em estoque, culminando na desistência do pedido. Outro fator limitador é a possibilidade de a série histórica em questão ter sido influenciada pela limitação de recursos orçamentários dos últimos anos, o que pode ter provocado uma oscilação anormal na demanda de peças de reposição no período em análise.

Como sugestão de estudos futuros, sugere-se: (i) verificar a aplicação de outros métodos que atualmente vêm sendo abordados na literatura; (ii) estudar a implementação de diferentes métricas de precisão, como o MASE (HYNDMAN; KOEHLER, 2006), para a mensuração dos erros das previsões no escopo funcionamento do SINGRA; e (iii) dar continuidade a este estudo para evidenciar a qualidade das previsões realizadas pelos métodos AES, Croston e SBA para a amostra de 276 itens de sobressalentes selecionada.

\section{REFERÊNCIAS BIBLIOGRÁFICAS}

[1] WILLEMAIN, T. R., SMART, C. N., SCHWARZ, H. F. A new approach to forecasting intermittent demand for service parts inventories. International Journal of

Forecasting, v. 20, n. 3, p. 375-387, jul-set. 2004. Disponível em: $<$ https://doi.org/10.1016/S0169-2070(03)00013-X>. Acesso em: 10 set. 2018.

[2] SYNTETOS, A.A.; BOYLAN, J. E; CROSTON, J. D. On the categorization of demand patterns. Journal of the Operational Research Society, v. 56, n.5, p. 495-503, mai. 


\section{SPOLM2019}

XIX SIMPÓSIO DE PESQUISA OPERACIONAL E LOGÍSTICA DA MARINHA RIO DE JANEIRO, RJ, BRASIL - 06 A 08 DE NOVEMBRO DE 2019

2005. Disponível em: < https://doi.org/10.1057/palgrave.jors.2601841>. Acesso em: 01 set. 2018 .

[3] KOURENTZES, N. On intermittent demand model optimisation and selection. International Journal of Production Economics, v. 156, p. 180-190, 2014.

[4] SYNTETOS, A. A.; BOYLAN, J. E. On the variance of intermittent demand estimates. International Journal of Production Economics, v. 128, n. 2, p. 546-555, dez. 2010.

[5] SYNTETOS, A. A.; BABAI, M. Z.; GARDNER, E. S. Forecasting intermittent inventory demands: simple parametric methods vs. bootstrapping. Journal of Business Research, v. 68, n. 8, p. 1746-1752, ago. 2015. Disponível em: <https://doi.org/10.1016/j.jbusres.2015.03.034>. Acesso em: 01 out. 2018

[6] MAKRIDAKIS, S.; WHEELWRIGHT, S. C.; HYNDMAN, R. J. Forecasting Methods and Applications. 3 ed. New York: John Wiley \& Sons, 1998.

[7] BRASIL. Marinha do Brasil. Secretaria-Geral da Marinha. Normas para Execução do Abastecimento (SGM-201). 6. Revisão. Brasília, 2009.

[8] BRASIL. Marinha do Brasil. Diretoria de Abastecimento da Marinha. Manual do Sistema de Informações Gerenciais do Abastecimento. Rio de Janeiro, 2007.

[9] PELLEGRINI, F. R.; FOGLIATTO, F. S. Passos para Implantação de Sistemas de Previsão de Demanda - Técnicas e Estudo de Caso. Revista Produção, v. 11, n. 1, p. 43-64, nov. 2001. Disponível em: <http://www.scielo.br/pdf/prod/v11n1/v11 n1a04>. Acesso em: 10 set. 2018.

[10] SYNTETOS, A. A.; BOYLAN, J. E. The accuracy of intermittent demand estimates. International Journal of Forecasting, v. 21, n. 2, p. 303-314, abr-jun. 2005. Disponível em: $<$ https://doi.org/10.1016/j.ijforecast.2004.10.001>. Acesso em: 10 set. 2018.

[11] WALLSTRÖM, P.; SEGERSTEDT, A. Evaluation of forecasting error measurements and techniques for intermittent demand. International Journal of Production Economics, v. 128, n. 2, p. 625-636, dez. 2010.

[12] BABAI, M. Z.; ALI, M. M.; NIKOLOPOULOS, K. Impact of temporal aggregation on stock control performance of intermittent demand estimators: Empirical analysis. Omega, v. 40, n. 6, p. 713-721, dez. 2012. Disponível em: <https://doi.org/10.1016/j.omega.2011.09.004> Acesso em: 20 set. 2018.

[13] PETROPOULOS, F.; KOURENTZES, N.; NIKOLOPOULOS, K. Another look at estimators for intermittent demand. International Journal of Production Economics, v. 181, p. 154-161, 2016.

[14] CROSTON, J. D. Forecasting and stock control for intermittent demands. Operational Research Quarterly, v. 23, n. 3, p. 289-303, set. 1972. Disponível em: $<$ https://www.jstor.org/stable/3007885>. Acesso em: 01 set. 2018.

[15] BOYLAN, J. E.; SYNTETOS, A. A.; KARAKOSTAS, G. C. Classification for forecasting and stock control: a case study. Journal of the Operational Research Society, v. 59, n. 4, p. 473- 
481, abr. 2008. Disponível em: <https://doi.org/10.1057/palgrave.jors.2602312> Acesso em: 10 set. 2018.

[16] SYNTETOS, A. A.; BOYLAN, J. E. On the bias of intermittent demand estimates. International Journal Production Economics, v.71, n. p. 457-466, 2001.

[17] TEUNTER, R. H.; SANI, B. On the bias of Croston's forecasting method. European Journal of Operational Research, v. 194, n. 1, p. 177-183, abr. 2009. Disponível em: $<$ https://doi.org/10.1016/j.ejor.2007.12.001>. Acesso em: 10 set. 2018.

[18] MAKRIDAKIS, S.; SPILIOTIS, E.; ASSIMAKOPOULOS, V. The M4 Competition: Results, findings, conclusion and way forward. International Journal of Forecasting, v. 34, p. 802808, out-dez. 2018. Disponível em: <https://doi.org/10.1016/j.ijforecast.2018.06.001>. Acesso em: 01 out. 2018.

[19] EAVES, A. H. C. Forecasting for the ordering and stockholding of consumable spare parts. 2002. $372 \mathrm{f}$. Tese (Doutorado em Psicologia) -Department of Management Science, The Management School, Lancaster University. 2002. Disponível em: $<$ http:// www.andalus-solutions.com/PhD\%20Thesis\%20(Eaves).pdf $>$. Acesso em: 01 out. 2018.

[20] SYNTETOS, A. A.; BOYLAN, J. E. On the stock control performance of intermittent demand estimators. International Journal Production Economics, v. 103, n. 1, p. 3647, set. 2006.

[21] TEUNTER, R. H.; DUNCAN, L. Forecasting intermittent demand: a comparative study. Journal of the Operational Research Society, v. 60, n. 3, p. 321-329, mar. 2009. Disponível em: < https://doi.org/10.1057/palgrave.jors.2602569>. Acesso em: 01 set. 2018.

[22] HYNDMAN, R. J.; KOEHLER, A. B. Another look at measures of forecast accuracy. International Journal of Forecasting, v. 22, p. 679-688, 2006. Disponível em: <http:// eva.fcea.edu.uy/pluginfile.php/109034/mod_resource/content $/ 0 / 2006$ Hyndman_Predicc.pdf $>$. Acesso em: 01 out. 2018.

[23] SYNTETOS, A. A. A note on managing lumpy demand for aircraft spare parts. Journal of Air Transport Management, v. 13, n. 3, p. 166-167, mai. 2007. Disponível em: $<$ https://doi.org/10.1016/j.jairtraman.2007.01.002>. Acesso em: 10 set. 2018.

[24] PRODANOV, C. C.; FREITAS, E. C. Trabalho Científico: Métodos e Técnicas da Pesquisa e do Trabalho Acadêmico. 2. ed. Rio Grande do Sul: Feevale, 2013.

[25] GIL, A. C. Métodos e técnicas de pesquisa social. 6. ed. São Paulo: Atlas, 2008.

[26] YIN, R. Estudo de caso: planejamento e métodos. 2. ed. Porto Alegre: Bookman, 2001. As Referências Bibliográficas devem seguir as normas da ABNT/NBR 6023. 
iEntrevista concedida pelo Gestor da Cadeia de Sobressalentes do SAbM. Entrevista I [out.2018]. Entrevistador: Autor. Rio de Janeiro, 2018.

iiEntrevista concedida pelo Encarregado do Apoio Logístico Integrado do SAbM. Entrevista II [out.2018]. Entrevistador: Autor. Rio de Janeiro, 2018. 